\title{
The use of direct composite resin to close maxillary midline diastema complementary to orthodontic treatment.
}

\author{
Gerardo Durán ${ }^{1 *}$, Francisca Vivar ${ }^{1}$, José Tisi ${ }^{1}$, Ismael Henríquez ${ }^{1}$
}

1. Facultad de Ciencias de la Salud, Universidad Arturo Prat, Iquique, Chile.

* Correspondencia autor. Gerardo Durán | E-mail: Gerardo.duran@unap.cl |Address: Avenida Arturo Prat 2120, Iquique, Chile. Universidad Arturo Prat. | Phone Number: +569 72115778

Trabajo recibido el 20/09/2018.

Aprobado para su publicación el 27/11/2018

\begin{abstract}
The maxillary midline diastema is a common esthetic problem in patients. Assess and diagnose may be challenging due to the many factors that influences the alteration of tooth eruption process and tooth shape. Due to the various advantages and the reversibility of treatments, composite resins are today a suitable aesthetic and functional option, even so, its management and technique require a trained and experienced clinician in order to obtain an optimal outcome. The following report, is a clinical case of a 21-year old female with a main complaint of a maxillary midline diastema produced by the alteration in tooth shape and size of both upper central incisors, which was closed and corrected with direct composite resins.

KEY WORDS:

Maxillary midline diastema, Diastema closure, Composite resin.
\end{abstract}

Rev. Clin. Periodoncia Implantol. Rehabil. Oral Vol. 12(2); 106-108, 2019

\section{INTRODUCTION}

Nowadays, dental and facial appearance is of great concern for clinicians in the field of restorative dentistry and prosthodontics, as well as for patients ${ }^{(1)}$.

Aesthetic problems are one of the most common reasons for consultation in dentistry practices, likely because issues related to appearance can influence many aspects of a patient's life, including his or her personality, physical attractiveness, work, and human relationships ${ }^{(2,3)}$. The presence of diastemas in the upper central incisors is one of the main aesthetic concerns for patients during dental appointments ${ }^{(4)}$. The maxillary midline diastema (MMD) is defined as a space greater than $0.5 \mathrm{~mm}$ between both mesial surfaces of the two central incisors ${ }^{(5)}$. It has a multifactorial etiology, and it can result from either hereditary or developmental factors and disorders. Among these, the most common are the persistence of the labial frenum, the absence of anterior teeth, and the presence of conoid lateral teeth, unerupted supernumeraries, lingual interposition habits, occlusal alterations, and macroglossia ${ }^{(6,7)}$.

There are several clinical therapies involving multidisciplinary approaches that help resolve an $\mathrm{MMD}^{(8)}$. These include the management of this alteration via orthodontic resolutions combined with surgical techniques that involve the resection of the labial frenum or restorative dentistry through rehabilitation using such materials as composite-based resins or ceramics ${ }^{(9)}$

The following case report examines a systematic step-by-step method for treating an MMD after an orthodontic treatment using stratified direct composite resins.

\section{CLINICAL REPORT}

A 21-year-old female patient was directed to one of the author's private practices presenting with an MMD after the completion of orthodontic treatment. An exhaustive clinical examination was performed, and the maintenance of orthodontic appliances and diastema between tooth 1.1 and 2.1 were noted. This included morphological and altered tooth proportions in both maxillary central incisors (Figure 1).

A complete analysis of tooth proportions was performed, and the initial height and width of both upper central incisors was determined. A therapeutic proposal was developed based on digital treatment planning, which established the ideal proportions for tooth 1.1 and 2.1. A conservative treatment with composite resin was possible, and the patient consented to proceed with the therapeutic approach. Therefore, the removal of the orthodontic appliances for tooth 1.1 and 2.1 was requested in order to execute the treatment.

An initial color test was performed using composite resin masses that would be used to restore both central incisors (Figure 2). Once the color

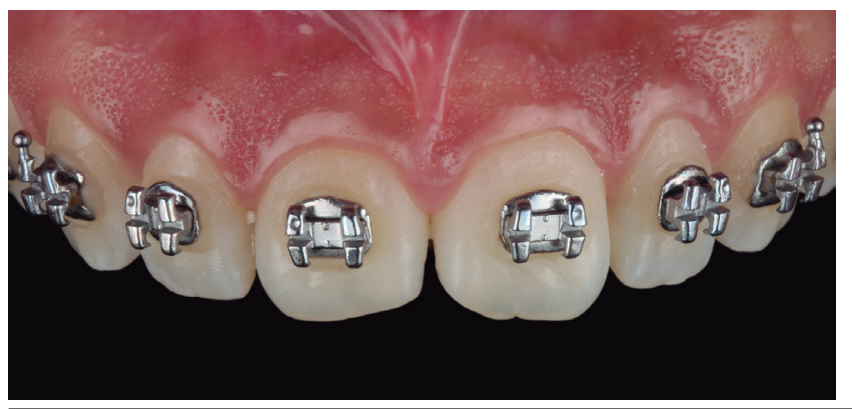

Figure 1. Initial situation. Patient presented with orthodontic appliances.

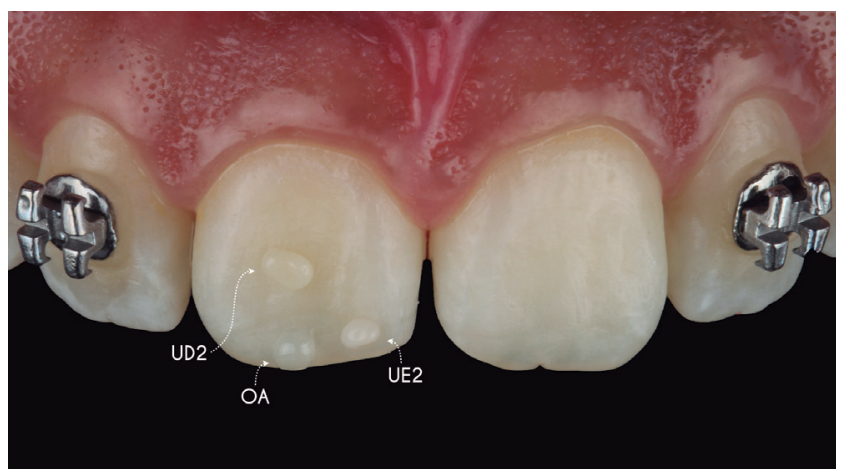

Figure 2. Composite resin color selection.

selection was made, it was decided to perform a restorative test. This procedure was done without including any kind of adhesive system such that the correct integration of shape and color matching the restorations could be verified in advance, allowing easy removal of the resins (Figure 3).

Under relative isolation with a rubber dam, the adhesive technique was performed on tooth 1.1. After the surface was cleaned with pumice, the enamel was etched with $37 \%$ phosphoric acid (Ultra-etch, UltraDent Products, Inc.) for 30 seconds (Figure 4), rinsed profusely with water, and the tooth's surface was dried. A thin layer of a two-step etch-and-rinse adhesive system (OptiBond S, Kerr Corporation, Orange, CA, USA) was then applied, air-thinned, and light-cured for 20 seconds. 


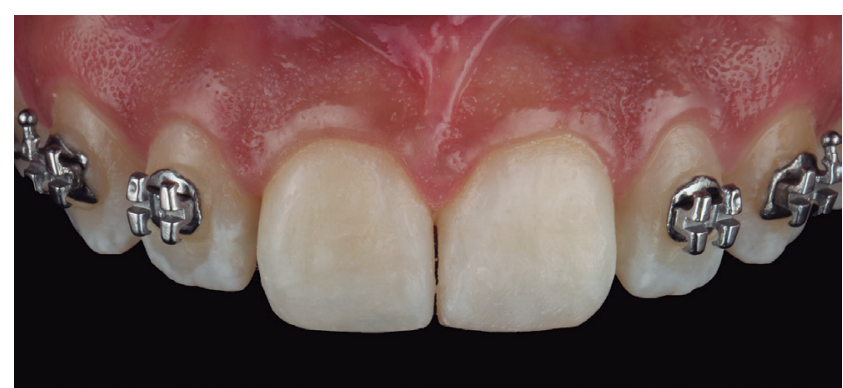

Figure 3. Restorative test without bonding technique. In this case, it can be appreciate that color match is acceptable, and diastema closure is possible.

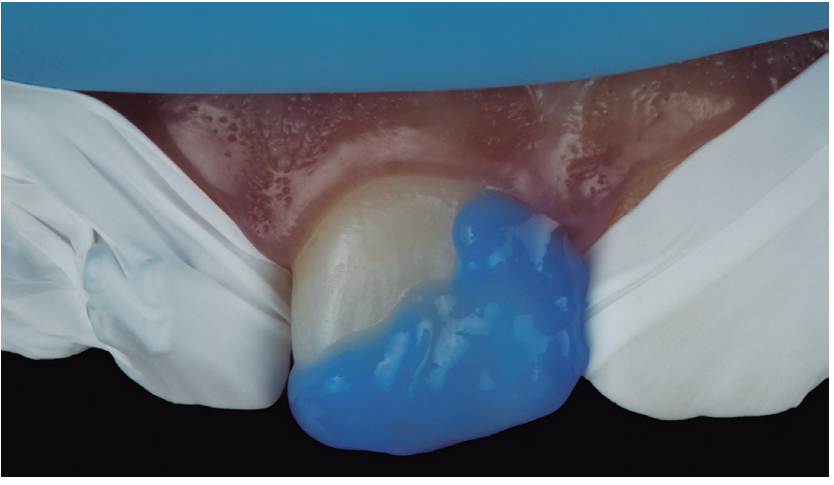

Figure 4. Bonding technique. Under partial isolation, application of bonding procedures are easy to perform. Initial step showing $37 \%$ phosphoric acid application in enamel. Neighboring tooth must be protected with teflon tapes

Once the enamel's surface was conditioned, a high reflective index composite resin was carefully applied using a free-hand technique to restore the proximal and palatal areas of the enamel (UE2, ENA Hri, Micerium SPA, Genova, Italy). This was then light-cured for 40 seconds. At this point, the clinician left enough space for the incorporation of a smal amount of opalescent resin in the incisal third (OBN, ENA Hri, Micerium SPA, Genova, Italy) and the enamel for the vestibular aspect of the tooth (UE2, ENA Hri, Micerium SPA, Genova, Italy).

After the restoration of tooth 1.1 was complete, the restoration of tooth 2.1 proceeded using the same approach. The clinician used small mylar strips during polymerization of the proximal composite resin layer in order to prevent bonding between both restorations.

The finishing and polishing procedure initially began with shaping the macro-morphology of the incisors using medium-sized aluminum oxide grit discs (Sof-Lex XT Medium, 3M ESPE). The vertical micro-anatomy was crafted using multi-laminated carbide burs. Horizontal micro-anatomy was not performed in this case because the patient had no clear microtexture marked by periquematis. Finally, the polishing procedure was carried out using diamond and aluminum oxide three-step pastes (ENA

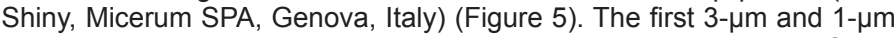
diamond pastes were applied sequentially with goat hair brushes (Shiny $A$ and B, Micerium SPA, Italy), and then the aluminum oxide paste was applied with cotton felt (Shiny C, Micerium Spa, Italy). Between each step, the clinician tried to clean the tooth surface with a gauze soaked in alcohol to eliminate oils contained in the polishing pastes.

Images of immediate control and the final outcome with a 10-month control are shown in figure 6 and 7 , respectively.

\section{DISCUSSION}

This clinical case describes a simple resolution for morphological alterations in both maxillary central incisors, which can produce an MMD. Composite resins were used in order to achieve harmony, proportionality, and aesthetics in the anterior tooth after orthodontic treatment.

From the clinical aspect, there are controversies about performing labial frenectomy and its indication when closing MMD. A complete evaluation of the shape, size and position must be done, which is in most of the cases performed after eruption of the permanent canines, since these can cause a spontaneous space closure during eruption. Frenectomy by itself is not recommended as the only treatment since it is indicated when an initial orthodontic treatment cannot close fully the diastema, or when the insertion is present in the palatal aspect(11). In this particular case,

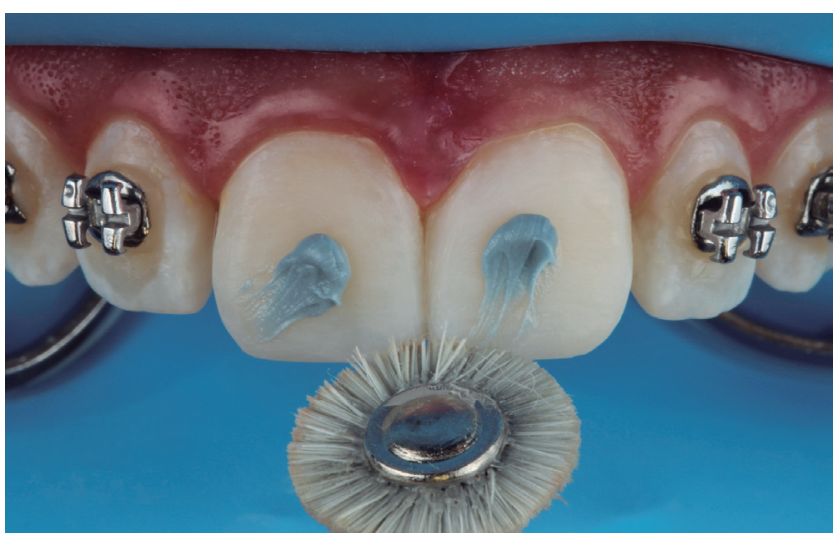

Figure 5. First polishing paste with diamond pastes of 1 micron. The application must be performed with hair coat wheel brushes.

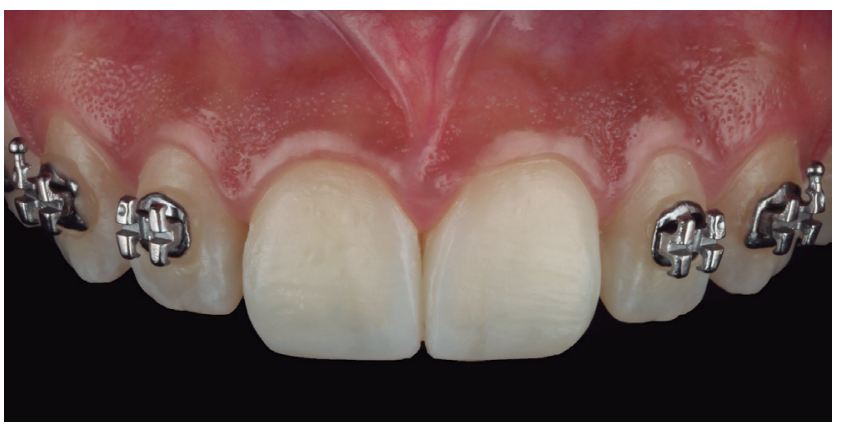

Figure 6. Immediate control after finishing and polishing procedure.

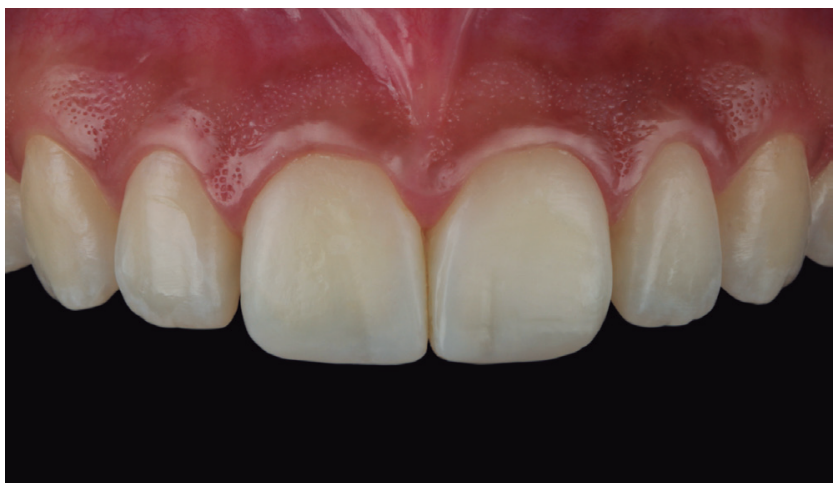

Figure 7. 10-month control image. Correct color and shape integration can be appreciated.

different shapes and proportion of the central incisors produce the MMD, and frenectomy is not indicated to correct this alteration ${ }^{(10)}$

One therapeutic restorative approach reported in the literature could be achieved using ceramic veneers or ceramic fragments ${ }^{(12)}$. These cases can be executed when there is vestibular enamel compromised tissue. Furthermore, this approach has the advantage that the mesial contact point can be controlled during the indirect method outside of the mouth before cementation. Optimal contact points can be obtained in the working model when compare with direct composite resins.

Closing an MMD using orthodontics may be achieved in many cases without the need for restorations when the morphology of the teeth and proportions are adequate ${ }^{(13)}$. Even so, in this particular clinical case, the height and width ratio was not correct, so orthodontics by itself could not solve the esthetic problem that this patient presented with. Trying to close the MMD could leave insufficient space to restore correct dental proportions in both central incisors.

Restorations with direct composite resins have clear advantages. For example, there is no need for tooth preparations when compared with laminate veneers, since direct composite resin restorations are a more conservative approach. Furthermore, there is a chance to repair the composite resin restorations in case they are dislodged or delaminated because the treatment is reversible. We emphasize that if the clinician selects a restorative treatment based on ceramic veneers that includes 
tooth preparation, there is no possibility to recover the lost enamel like one can with composite resins because of the lack of tooth preparation. There are fewer clinical appointments compared to any other indirect method for restoration. Finally, the final outcome depends only on the clinician ${ }^{(14)}$

One possible disadvantage identified during the analysis of this case is the necessity for re-polishing composite resins. It has been reported that nanohybrid composites like those used in this case (Enamel Hri, Micerium Spa, Genova, Italy) have high polishing gloss unit values, but this is lost over time due to many dietary, functional, and parafunctional factors ${ }^{(15)}$. Another disadvantage is that the free-hand restorative technique is difficult without using a guide or silicone index from a previous diagnostic wax-up. As such, clinical expert must be highly trained in order to provide a successful long-lasting restoration.

The use of a restorative test allows the visualization of the final outcome in advance in a hydrated situation previously isolation and the adhesive technique. Using this simple approach, we can determine whether the restorative approach will have the desired results. We should remember, however, that very sharp angles must be rounded in order to obtain a visually seamless transition between the restoration and the enamel. Furthermore, the enamel surfaces must be cleaned before bonding to obtain better bond strength.

\section{SUMMARY}

Direct composite restorations to close an MMD after an orthodontic treatment may be a simple and conservative clinical solution that yields optimal aesthetic results. The authors suggest making an initial restorative test before clinical execution in order to pre-visualize the color and quality of shape integration of the restoration.

\section{DECLARATION OF INTEREST}

The authors declare they have not conflict of interests.

\section{ACKNOWLEDGEMENTS}

The authors want to express their acknowledgement to Prof. Dr. Bruno Caroca who did the previous orthodontic treatment and trusted his patient to our team in order to deliver the restorative treatment. Also, we personally thank the Director of the Vice Rectory of Research, Innovation and Postgraduate, and the Health Sciences Faculty of Universidad Arturo Prat, Iquique, Chile, for encourage our research work.

\section{References}

1. Mehl C, Wolfart S, Vollrath $\mathrm{O}$, Wenz $\mathrm{HJ}$, Kern M. Perception of dental esthetics in different cultures. Int J Prosthodont. 2014;27(6):523-529.

2. Işiksal E, Hazar S, Akyalçin S. Smile esthetics: perception and comparison of treated and untreated smiles. Am J Orthod Dentofacial Orthop. 2006:129:8-16.

3. Beall AE. Can a new smile make you look more intelligent and successful? Dent Clin North Am. 2007;51:289-297.

4. Chu $\mathrm{CH}$. Chang CF. Jin LJ. Trating a maxillary midline diastema in adult patients: A general dentist's perspective. J Am Dent Assoc. 2011:142(11):1258-1264.

5 . Keene $\mathrm{H}$. Distribution of diastemas in the dentition of man. Am J Phys Anthropol. 1963;21:437-441.

6. Silva LC, Matos C, Oliveira T, Melo P, Silva MJ. Encerramento de Diastemas. Revisão de conceitos teóricos a propósito de un caso clínico. Rev Port Estomatol Cir Maxilofac. 2008;49:133-139.

7. Oesterle LJ. Shellhart WC. Maxillary midline diastemas: a look at the causes. J Am Dent Assoc. 1999;130(1):85-94

8. Huang WJ. Creath CJ. The midline diastema: a review of its etiology and treatment. Pediatr Dent. 1995;17(3):171-179.

9. Romero MF, Babb CS, Brenes C, Haddock FJ. A multidisciplinary approach to the management of a maxillary midline diastema: A clinical report. J Prosthet Dent. 2018 119(4):502-505
10. Suter VG, Heinzmann AE, Grossen J, Sculean A, Bornstein MM. Does the maxillary midline diastema close after frenectomy?. Quintessence Int. 2014,45(1):57-66. 11. Gkantidis N, Kolokitha OE, Topouzelis N. Management of maxillary midline diastema with emphasis on etiology. J Pediatr Dent. 2008;32(4):265-272.

12. Miranda M, Olivieri K, Rigolin F, Basting R. Ceramic Fragments and metal-free full crowns: A conservative esthetic option for closing diastemas and rehabilitating smiles. Oper Dent. 2013;38(6):567-571.

13. Moffitt A, Raina J. Long-term bonded retention after closure of maxillary midline diastema. Am J Orthod Dentofacial Orthop. 2015;148(2):238-244.

14. Hwang S, Ha J, Jin M, Kim S, Kim Y. Diastema closure using direct bonding restorations combined with orthodontic treatment: a case report. Restor Dent Endod. 2012;37(3):165.

15. Kaizer M, de Oliveira-Ogliari A, Cenci M, Opdam N, Moraes R. Do nanofill or submicron composites show improved smoothness and gloss? A systematic review of in vitro studies. Dent Mater. 2014;30(4):e41-e78. 